\title{
Erratum to: Effect of allopurinol on cardiovascular incidence among hypertensive nephropathy patients: the Gonryo study
}

\author{
Hiroyuki Terawaki - Masaaki Nakayama - Emiko Miyazawa - Yaeko Murata • \\ Keisuke Nakayama - Masato Matsushima - Mariko Miyazaki · Hiroshi Sato • \\ Mitsuhiro Sato $\cdot$ Toshinobu Sato $\cdot$ Yoshio Taguma $\cdot$ Sadayoshi Ito
}

Published online: 18 January 2013

(C) Japanese Society of Nephrology 2013

\section{Erratum to: Clin Exp Nephrol \\ DOI 10.1007/s10157-012-0742-z}

In the original version of this article, the "Study contributors" was missing in the Acknowledgments and should have been included as follows.

Acknowledgments: This study was supported by a Grant from Astellas Pharm Inc. The authors express their special thanks to Ms. Makiko Nakayama for her assistance.

The online version of the original article can be found under doi:10.1007/s10157-012-0742-z.

H. Terawaki $(\bowtie) \cdot$ M. Nakayama

Department of Nephrology and Hypertension, Fukushima

Medical University School of Medicine, Fukushima, Japan

e-mail: terawaki@fmu.ac.jp

M. Nakayama - M. Matsushima - M. Miyazaki · H. Sato ·

T. Sato $\cdot$ S. Ito

Center for Advanced Integrated Renal Science, Tohoku

University Graduate School of Medicine, Sendai, Japan

E. Miyazawa · Y. Murata · K. Nakayama $\cdot$ M. Miyazaki $\cdot$ S. Ito

Department of Nephrology, Endocrinology and Vascular

Medicine, Tohoku University Graduate School of Medicine,

Sendai, Japan

\section{Matsushima}

Department of Clinical Research, The Jikei University School

of Medicine, Tokyo, Japan

H. Sato

Department of Clinical Pharmacology, Tohoku University

Graduate School of Pharmacology, Sendai, Japan

M. Sato · T. Sato · Y. Taguma

Kidney Center, Sendai-shakaihoken Hospital, Sendai, Japan
Study contributors: Yuji Yamaguchi (Japanese Red Cross Sendai Hospital), Katsuya Obara (Tohoku Kosai Hospital), Isao Kurihara (Tohoku Kosai Miyagino Hospital), Yasumichi Kinoshita and Kazuto Sato (Japanese Red Cross Ishinomaki Hospital), Jin Seino (Miyagi National Hospital), Akira Sugiura and Masahiro Miyata (Osaki Citizen Hospital), Kazuhisa Takeuchi (Koujinkai Central Hemodialysis Clinic), Kenji Nakayama and Naoki Akiu (Sendai City Hospital), and Tetsuya Otaka (Katta General Hospital). 